\title{
A Curious Umbilical Fistula: An Unexpected Onset of Urachal Mucinous Cystic Tumour
}

\author{
Filippo Petrelli ${ }^{*}$, Roberta Rossi ${ }^{1}$, Maurizio S Fianchini ${ }^{2}$, Luca Cardinali ${ }^{1}$, Daniele Marrelli ${ }^{3}$ and Cristina Marmorale ${ }^{1}$ \\ ${ }^{1}$ Department of Surgery, Polytechnic University of Marche Region, Ancona, Italy \\ ${ }^{2}$ Clinic of Surgery, "AUO Ospedali Riuniti" Hospital, Ancona, Italy \\ ${ }^{3}$ Department of General Surgery and Surgical Oncology, University of Siena, Italy
}

\begin{abstract}
Background: The urachus is an embryonic remnant, which is obliterated in most of the adults. Residual of it persists in $32 \%$ of adults and neoplasms, which can arise from this structure, are extremely rare. These are usually diagnosed through incidental findings or for urinary symptoms. Their natural history is characterized by an early metastatization through the peritoneal cavity. Due to their rarity, there are no unanimous consensus for their management and therapy. Radical excision, associated or not with intraperitoneal hypertermic chemotherapy linked to cytoreductive surgery, was suggested over time.
\end{abstract}

Case report: A black woman came to our attention for a gelatinous secretion from the umbilicus. After a MRIstudy, a parietal neoformation of the urachal remnants was diagnosed. This neoformation revealed a cutaneous fistulization and it was excised surgically. Its pathologic exam described the presence of a cystic mucinous urachal tumor. During the follow-up, a cytoreductive surgery (CRS) and hyperthermic intraperitoneal chemotherapy (HIPEC) was performed, due to the occurence of free intraperitoneal liquid and multiple nodules on the abdominal parenchimas, After the last surgery, the patient underwent radiological and serological follow up and, after 6 months, there are no evidence of pathological recurrence.

Discussion and conclusion: In the diagnostic pathway, the MRI has a pivotal role for the study of the peritoneal cavity. Surgically, the complete excision is preferred. The role of the conventional chemotherapy is still under debate, however CRS in association with HIPEC, is certainly useful.

Keywords: Urachus; Umbilical fistula; HIPEC; Urachal carcinoma; Abdominal wall

\section{Introduction}

The urachus is an embryonic remnant of the urogenital sinus and the allantois [1]. It is a median structure which links the apex of the bladder with the umbilicus [2] and it is made up of three layers: an external muscular layer, connective tissue and a central lumen covered with a transitional or cuboidal epithelium [3,4]. This structure is progressively obliterated, becoming the median umbilical ligament in the adult [4]. Remnants of this structure persists in around $32 \%$ of adults and are usually located at the bladder dome, less frequently in its lateral and posterior walls, or in the space of Retzius $[5,6]$. Urachal neoplasms are extremely rare and, although the surface epithelium of this structure is mainly transitional, in most cases, tumours originate from glandular epithelium, as they are adenocarcinomas $[2,4,7,8]$. However, these neoplasms are still highly rare $[3,7,9,10]$ and their onset is more frequent in men between 40 and 70 [11-15]. The most recurring symptom of this neoplasm is hematuria, roughly associated with irritative symptoms such as dysuria, urinary urgency, suprapubic pain and vesical tenesmus $[11,16]$. Different anatomopathological classifications of these neoplasms have been proposed over time without reaching unanimous consensus. The latest proposed classification divides these tumours in glandular, nonglandular and mixed tumours. The main glandular tumours are adenomas, mucinous tumours of low malignant potential and adenocarcinomas, whether cystic or not. Nonglandular tumours, instead, may be urothelial, squamous, neuroendocrine and mixed type [4]. Many staging systems have been proposed as well over time $[14,17,18]$. The most employed system, and the one which has revealed greater predictive values, with regard to survival rate after surgery, is the Sheldon system [19]. Prognosis linked with malignization of the urachus is often poor. This is usually due to a delayed diagnosis and early peritoneal metastatization, which can lead to pseudomyxoma peritonei (PMP). PMP linked to urachal tumour is defined as: the presence of lower abdomen tumour located at the urachus or mucosuria; the observation of the tumour connected with the umbilicus and the urinary bladder during surgery; the definite urachal disease or history of it, without any other pathologic origin during the whole disease course [20]. The latest and most important studies show a 5 -year survival rate between $45 \%$ and $50 \%$ of all patients $[3,4,9,14,19,21]$. Predictive and independent indicators are the diffusion of tumour in adjacent organs, the presence of metastasis and residual disease after surgery. Metastatic disease occurs between 32 and $39 \%$ of patients, especially in lungs, bone and liver. Further recurring metastatization sites are lymph nodes and peritoneal system [14,19,21].

There is no unanimous consensus concerning the most accurate diagnostic - therapeutic process, due to the rarity of this pathology [3]. In most cases, the urachus excision has been carried out with, at times, radical or partial cystectomy [19]. The establishment of a chemotherapy regimen is not generally undertaken since its role is not well-defined [3]. Nevertheless, adjuvant or neoadjuvant chemotherapy has been

*Corresponding author: Dr. Filippo Petrelli, Department of Surgery, Polytechnic University of Marche Region, Ancona, Via S. Maria 35/B, 60015 Falconara Marittima (AN), Italy, Tel: 393334718217; Fax: 390719188182; E-mail: petrellifil@gmail.com

Received February 25, 2018; Accepted March 19, 2018; Published March 22 2018

Citation: Petrelli F, Rossi R, Fianchini MS, Cardinali L, Marrelli LD, et al. (2018) A Curious Umbilical Fistula: An Unexpected Onset of Urachal Mucinous Cystic Tumour. J Cancer Sci Ther 10: 60-63. doi:10.4172/1948-5956.1000518

Copyright: (C 2018 Petrelli F, et al. This is an open-access article distributed under the terms of the Creative Commons Attribution License, which permits unrestricted use, distribution, and reproduction in any medium, provided the original author and source are credited. 
suggested, with combined regimen of 5 -fluorouracil and cisplatin in some metastatic disease cases $[4,22]$. Cytoreductive surgery (CRS) and hyperthermic intraperitoneal chemotherapy (HIPEC) with mitomycin and oxaliplatin have been proposed in pseudomyxoma peritonei cases [20-23].

\section{Case Report}

A 45-year-old African woman came to our attention owing to a spontaneous leak of gelatinous material from the umbilicus. The patient reported positive history of hysterectomy, performed due to postpartum haemorrhage with fibromatous uterus and arterial hypertension. The clinical examination of the patient was negative, and the laboratory evaluations did not reveal any abnormality. Therefore, the magnetic resonance imaging (MRI) with contrast, revealed a nodular formation at paraumbilical and, largely, at intra-abdominal site. This formation revealed multiple septum, $5 \times 3 \mathrm{~cm}$ in diameter with liquid-corpuscular contents, connected with the skin through the rectus abdominis. Furthermore, this formation seemed to be strictly connected to some ileal loops. There was no evidence of further lesions at the main abdominal parenchymas. However, the presence of effusion between intestinal loops proved to be positive (Figure 1).

It was therefore decided to proceed with an exploratory laparotomy and the excision of the mass described in the radiological examination. During surgery, it was observed as follows: presence of mucoid material especially in the pelvis, omental adhesions with neoformation, which revealed infiltrative aspects and seemed broken (Figure 2).

It was thus decided to remove the mass in continuity with the umbilicus, the omentum and the adjacent muscle fascia, the urachus in toto and its insertion on the bladder. In agreement with the urologists, it was chosen not to perform a cystectomy, neither partial nor radical, for there was no evident invasion of the bladder. Histological examination of the lesion revealed the presence of mucinous cystic tumour, arising from the urachus, of low malignant potential and with negative microscopic margin. The patient was therefore referred to radiological follow-up, in agreement with our oncologist. A cystoscopy was performed 6 weeks after the first surgery, due to the appearance of a single episode of hematuria, which did not reveal neoformations at the bladder wall or presence of mucus in the bladder. Two months after the last surgery, the patient got an MRI, which showed minimum perihepatic hypogastric film; and dosage of tumour markers highlighted a light increase in CEA levels. It was therefore performed

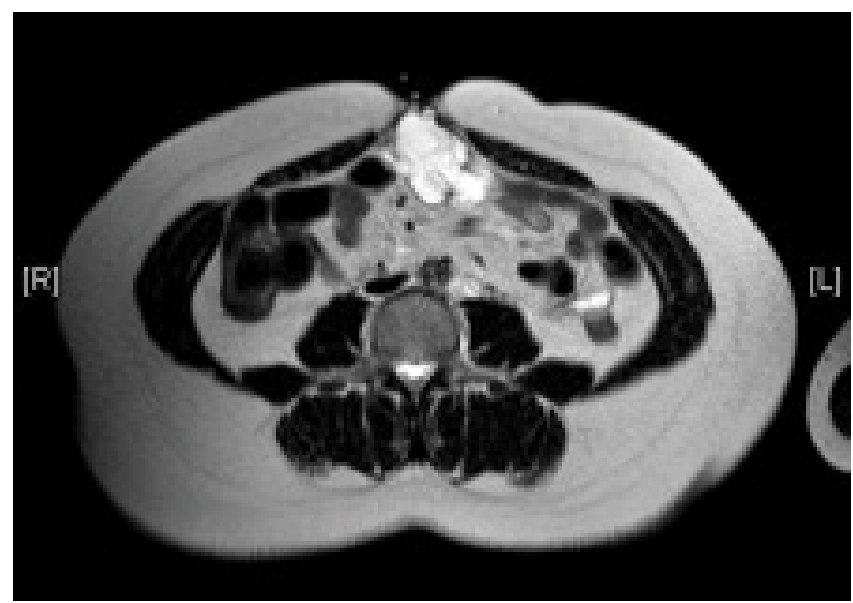

Figure 1: MRI Image of fistulised neoformation through the skin.

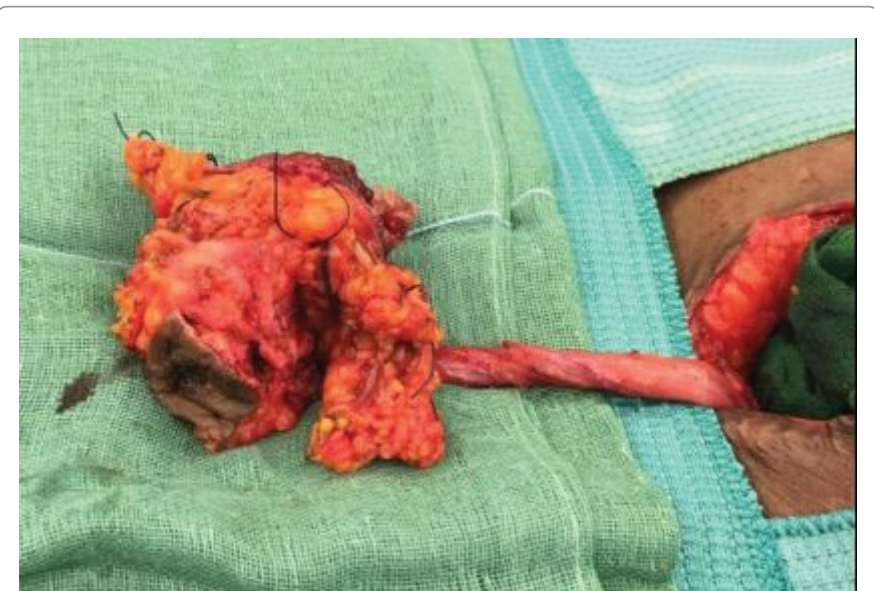

Figure 2: Surgical specimen not yet dissected.

an exploratory laparoscopy surgery, in order to evaluate the origin of the effusion. During surgery, which was performed 10 weeks after the first one, presence of millimetre-sized nodules at pelvic peritoneum and scarce effusion at pelvis and perihepatic space level, were observed. Withdrawals of intra-abdominal fluid were carried out during this procedure, but they were not diagnostic. The risk of carcinoma was very high because of the perforation and the presence of mucous in the peritoneal cavity.

That is why, two months later, the patient got an MRI, which described the appearance of a new nodular formation at the right subdiaphragmatic space and the left hypocondrium with minimum intra-abdominal film. Subsequently, the patient got a staging thoracicabdominal CT as well. CT scan highlighted a nodular formation at apical segment in the left lower pulmonary lobe with a no unequivocal interpretation, so worthy of follow-up, and it confirmed the presence of those lesions described in the resonance. Therefore, the patient underwent peritoneal cytoreductive surgery (CRS) with the addition of HIPEC at Policlininic "Santa Maria alle Scotte" in Siena. The patient got both further dosage of tumour markers, as preoperative evaluation, revealing an additional high increase in CEA levels, and thoracicabdominal CT, showing a dimensional increase above $20 \%$ of peritoneal carcinosis multiple implants with pathological lymph nodes at right cardiophrenic angle. During the surgical procedure, disease localization at hepatic Glisson's capsule, hepatoduodenal and hepatogastric ligaments, right hemidiaphragm, gastric greater curvature, the greater omentum, the pelvic peritoneum, left hemidiaphragm, jejunal and distal ileals, their mesenteries and at the anterior rectal wall as well, emerged from the abdominal cavity exploration. The removal of all macroscopic disease localizations and the parietal peritoneum adjent to the interesting sites, omentectomy, colecystectomy and appendicectomy, and the removal of uterine remnant on the vagina was therefore carried out, without intestinal resections (Figures 3-5)

At the end of the surgery, HIPEC was performed with $190 \mathrm{mg}$ of cisplatin and $38 \mathrm{mg}$ of mitomycin, infused intraperitoneally for 60 minutes with closed-abdomen technique. Histological examination indicated mucinous adenocarcinoma with medium degree of differentiation (G2; CK20+, CDX2+, CK7, WT1), without neoplastic infiltration of the 8 examined lymph nodes. The patient is currently subjected to radiological follow-up without performing adjuvant chemotherapy. After 12 months from the last treatment, the patient is in good general clinical conditions, without reporting urinary or 
Citation: Petrelli F, Rossi R, Fianchini MS, Cardinali L, Marrelli LD, et al. (2018)A Curious Umbilical Fistula: An Unexpected Onset of Urachal Mucinous Cystic Tumour. J Cancer Sci Ther 10: 60-63. doi:10.4172/1948-5956.1000518

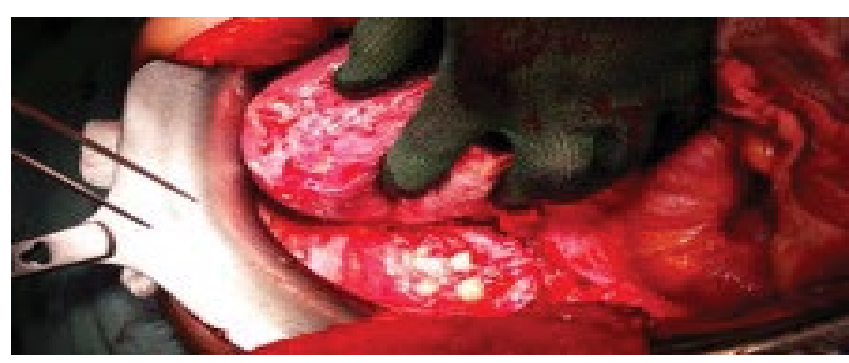

Figure 3: Nodularity at the lower face of the right hemidiaphragm.

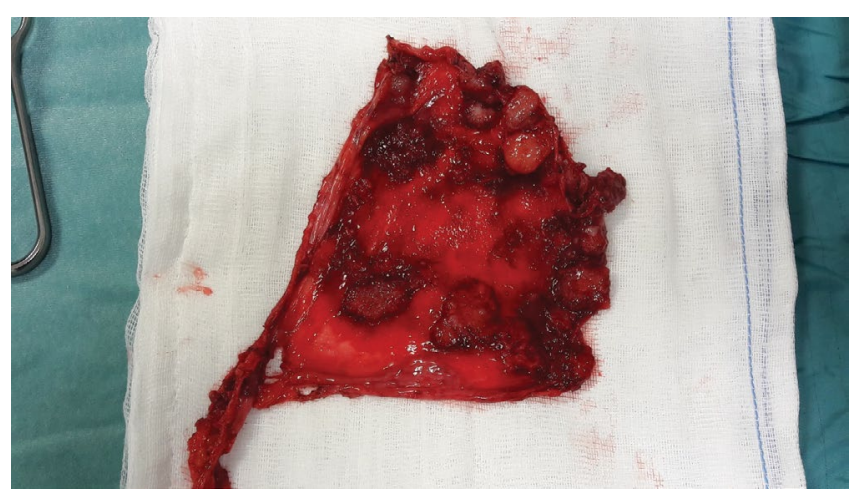

Figure 4: Parietal peritoneum of the lower face of the right hemidiaphragm after the removal.

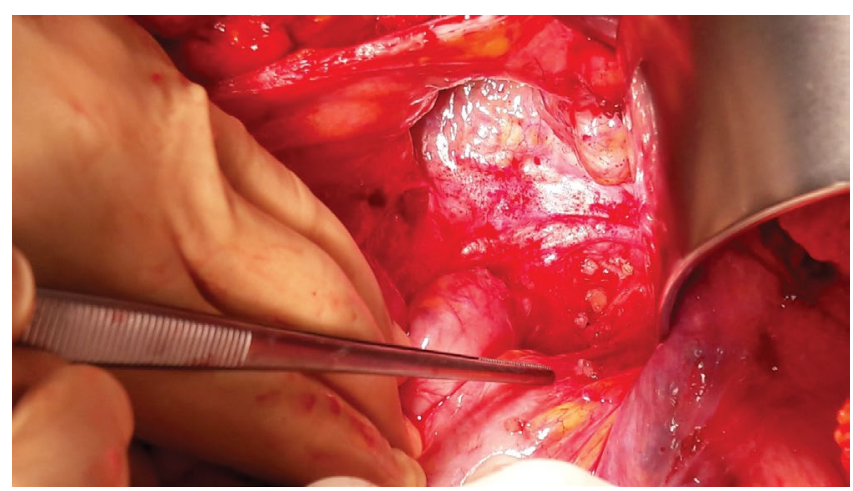

Figure 5: Parietal peritoneum of the lower face of the right hemidiaphragm after the removal.

abdominal symptoms. Furthermore, the radiological check-up is negative, as well as biohumoral markers, which are stable.

\section{Discussion}

Urachal mucinous cystic tumours are extremely rare $[7,8]$. This is the reason why there are no unequivocal indications or guidelines in literature with regard to the management of these cases. The magnetic resonance (MRI) has an important role for the diagnosis and stadiation of this disease as it can study soft tissues of the abdominal wall and it observes the presence of mucus in the peritoneal cavity, for it has been observed that these lesions tend to spread in every part of the peritoneum [14]. Generally, these neoplasms are diagnosed as occasional acknowledgment or after the appearance of urinary symptoms.

\section{Conclusion}

The peculiarity of our case is the atypical onset of the symptoms, arose through cutaneous fistulization rather than a bladder fistula, so without urinary symptoms. Furthermore, there is no univocal surgical strategy in case of not clear communication with the urinary apparatus, except for the en-bloc resection of the mass (more or less spread to neighbouring tissues). Moreover, the role of conventional chemo- and/or radiotherapy (mainly and exclusively performed in metastatic diseases) is not clear in literature. These are the reasons why a cautious strategy has been chosen with regard to the disease, treated whenever traced during the follow up, trying to obtain its radicality. For this purpose, in the light of good results achieved in literature [20], it has been decided to perform the cytoreductive surgery (CRS) and the hyperthermic intraperitoneal chemotherapy (HIPEC), in case of PMP associated with the urachal neoplasia.

\section{References}

1. Pasternak MC, Black JD, Buza N, Azodi M, Gariepy A (2014) An unexpected mass of the urachus: a case report. Am J Obstet Gynecol 211: 1-3.

2. Gopalan A1, Sharp DS, Fine SW, Tickoo SK, Herr HW, et al. (2009) Uracha carcinoma: a clinicopathologic analysis of 24 cases with outcome correlation. Am J Surg Pathol 33: 659-668.

3. Mylonas KS, P OM, Ziogas IA, El-Kabab L, Nasioudis D (2017) Malignant urachal neoplasms: A population-based study and systematic review of literature. Urol Oncol 35: 11-19.

4. Paner GP, Lopez-Beltran A, Sirohi D, Amin MB (2016) Updates in the Pathologic Diagnosis and Classification of Epithelial Neoplasms of Urachal Origin. Adv Anat Pathol 23: 71-83.

5. Blichert-Toft M, Koch F, Nielsen OV (1973) Anatomic variants of the urachus related to clinical appearance and surgical treatment of urachal lesions. Surgery, Gynecology Obstetrics 137: 51-54.

6. Schubert GE, Pavkovic MB, Bethke-Bedurftig BA (1982) Tubular urachal remnants in adult bladders. J Urol 127: 40-42.

7. Siefker-Radtke AO, Gee J, Shen Y, Wen S, Daliani D, et al. (2003) Multimodality management of urachal carcinoma: the M. D. Anderson Cancer Center experience. J Urol 169: 1295-1298.

8. Amin MB, Smith SC, Eble JN (2014) Glandular neoplasms of the urachus: a report of 55 cases emphasizing mucinous cystic tumors with proposed classification. Am J Surg Pathol Aug 38: 1033-1045.

9. Dhillon J, Liang Y, Kamat AM, Siefker-Radtke A, Dinney CP, et al. (2015) Urachal carcinoma: a pathologic and clinical study of 46 cases. Hum Patho 46: 1808-1814

10. Wang B, Li X, Ming S, Ma X, Li H, et al. (2016) Combined extraperitoneal and transperitoneal laparoscopic extended partial cystectomy for the treatment of urachal carcinoma. J Endourol 30: 280-285.

11. da Cruz CL, Fernandes GL, Natal MR, Taveira TR, Bicalho PA, et al. (2014) Urachal neoplasia: a case report. Radiologia brasileira 47: 387-388.

12. Yu JS, Kim KW, Lee HJ, Lee YJ, Yoon CS, et al. (2001) Urachal remnant diseases: spectrum of CT and US findings. Radiographics : a review publication of the Radiological Society of North America, Inc. 21: 451-461.

13. Koster IM, Cleyndert P, Giard RW (2009) Best cases from the AFIP: urachal carcinoma. Radiographics a review publication of the Radiological Society of North America, Inc. 29: 939-942.

14. Ashley RA, Inman BA, Sebo TJ, Leibovich BC, Blute ML, et al. (2006) Uracha carcinoma: clinicopathologic features and long-term outcomes of an aggressive malignancy. Cancer 107: 712-720.

15. Thali-Schwab CM, Woodward PJ, Wagner BJ (2005) Computed tomographic appearance of urachal adenocarcinomas: review of 25 cases. Eur Radiol Jan 15: $79-84$

16. Wang LL, Liddell H, Tanny ST, Norris B, Appu S, et al. (2016) Incidenta Finding of a Rare Urachal Pathology: Urachal Mucinous Cystic Tumour of Low Malignant Potential. Case Rep Urol 2016: 5764625. 
Citation: Petrelli F, Rossi R, Fianchini MS, Cardinali1, Marrelli LD, et al. (2018) A Curious Umbilical Fistula: An Unexpected Onset of Urachal Mucinous Cystic Tumour. J Cancer Sci Ther 10: 60-63. doi:10.4172/1948-5956.1000518

17. Pinthus JH, Haddad R, Trachtenberg J, Holowaty E, Bowler J, et al. (2006) Population based survival data on urachal tumors. J Urol 175: 2042-2047.

18. Sheldon CA, Clayman RV, Gonzalez R, Williams RD, Fraley EE (1984) Malignant urachal lesions. J Urol 131: 1-8.

19. Herr HW, Bochner BH, Sharp D, Dalbagni G, Reuter VE (2007) Urachal carcinoma: contemporary surgical outcomes. J Urol 178: 74-78.

20. Liu Y, Ishibashi H, Hirano M, Takeshita K, Mizumoto A, et al. (2015) Cytoreductive surgery plus hyperthermic intraperitoneal chemotherapy for pseudomyxoma peritonei arising from urachus. Ann Surg Oncol 22: 2799-805.
21. Bruins HM, Visser $O$, Ploeg $M$, Hulsbergen-van de Kaa CA, Kiemeney LA (2012) The clinical epidemiology of urachal carcinoma: results of a large, population based study. J Urol 188: 1102-1107.

22. Yanagihara $Y$, Tanji N, Miura N, Shirato A, Nishimura K, et al. (2013) Modified FOLFOX6 chemotherapy in patients with metastatic urachal cancer. Chemotherapy 59: 402-406.

23. Sugarbaker $\mathrm{PH}$ (1996) Peritonectomy procedures. Cancer Treatment Research 82: $235-253$ 